\title{
Characterization of Small Cu Grains Using the Conical Dark-Field Technique in the Transmission Electron Microscope
}

\author{
R. Hübner, ${ }^{*}$ H.-J. Engelmann, ${ }^{* *}$ and E. Zschech* \\ * Fraunhofer Institute for Non-Destructive Testing IZFP, Dresden Branch, Maria-Reiche-Strasse 2, \\ 01109 Dresden, Germany \\ ** GLOBALFOUNDRIES Dresden Module One LLC \& Co. KG, Wilschdorfer Landstrasse 101, \\ 01109 Dresden, Germany
}

On-chip $\mathrm{Cu}$ interconnects with dimensions significantly smaller than $100 \mathrm{~nm}$ are characterized by a changed microstructure, in particular by modified grain size distributions, grain orientations, and stress states. As a consequence, reliability-limiting degradation mechanisms such as electromigration and stress-induced voiding (SIV) might change their characteristics. For these reasons, advanced analytical techniques are necessary to control and to precisely monitor the $\mathrm{Cu}$ microstructure in narrow interconnect lines.

Conventionally, X-ray microdiffraction (micro-XRD) and electron backscatter diffraction (EBSD) have been applied for $\mathrm{Cu}$ microstructure analysis. However, the characterization of regions with multiple small grains requires advanced techniques. Conical Dark-Field (CDF) analysis in the TEM has the potential to provide complete grain orientation distributions. In a first step, centered darkfield images of a particular view of the specimen are recorded in a circular way for several tilt and rotation angles of the parallel electron beam. In a second step, spot diffraction patterns for every point of the view of interest are reconstructed. Based on the crystallographic structure of the polycrystalline material, the diffraction patterns are indexed [1].

As an example, Fig. 1 shows a bright-field image together with an inverse pole figure (IPF) map of a $\mathrm{Cu}$ thin film deposited onto a Ta diffusion barrier. To analyze a larger part of the $\mathrm{Cu}$ film, two independent $\mathrm{CDF}$ measurements were stitched together. The $\mathrm{Cu}$ film is predominantly $<111>$ oriented perpendicular to the wafer surface (not shown here). In addition, strong nano-twinning behavior with twin planes parallel to the wafer surface is observed (Fig. 1(b)). With the smallest twin lamella thicknesses of $5 \mathrm{~nm}$ in Fig. 1(b), the extremely high spatial resolution for grain orientation determination using the CDF technique in the TEM is confirmed [2].

The bright-field image of a $\mathrm{Cu} / \mathrm{low}-k$ interconnect stack after completing a stress migration reliability test is shown in Fig. 2(a). In this case, FIB target preparation was performed in such a way that the void formed during SIV-based degradation and the surrounding $\mathrm{Cu}$ grains are partially included in the TEM lamella. As shown in Fig. 2(b), $\mathrm{Cu}$ microstructure information is provided for the region next to the void formed at the edge of the via bottom, which is the location of the maximum stress gradient, where eventually the damage occurs. At the position of the small grains in the direct vicinity of the void energy-filtered TEM showed an increased oxygen concentration, which might be caused by oxide formation at the inner surface of the void. According to Fig. 2(b), the Cu grains in the wide line are characterized by a preferred $\langle 111>$ orientation relative to the wafer surface. This orientation was found to be not as resistant to SIV as Cu structures with random texture [3]. 
Although characterized by a high spatial resolution, the CDF method is associated with several challenges. Besides good TEM alignment, negligible specimen drift and beam-induced sample alterations, suitable parameter choice during automated data evaluation is crucial for successful orientation determination. A manual check of the obtained results is advisable. It is absolutely essential in the case of superimposing diffraction patterns.

References

[1] D. Dingley, Microchim. Acta 155 (200) 19.

[2] R. Hübner et al., AIP Conf. Proc. 1300 (2010) 39.

[3] E. Zschech et al., J. Appl. Phys. 106 (2009) 093711.
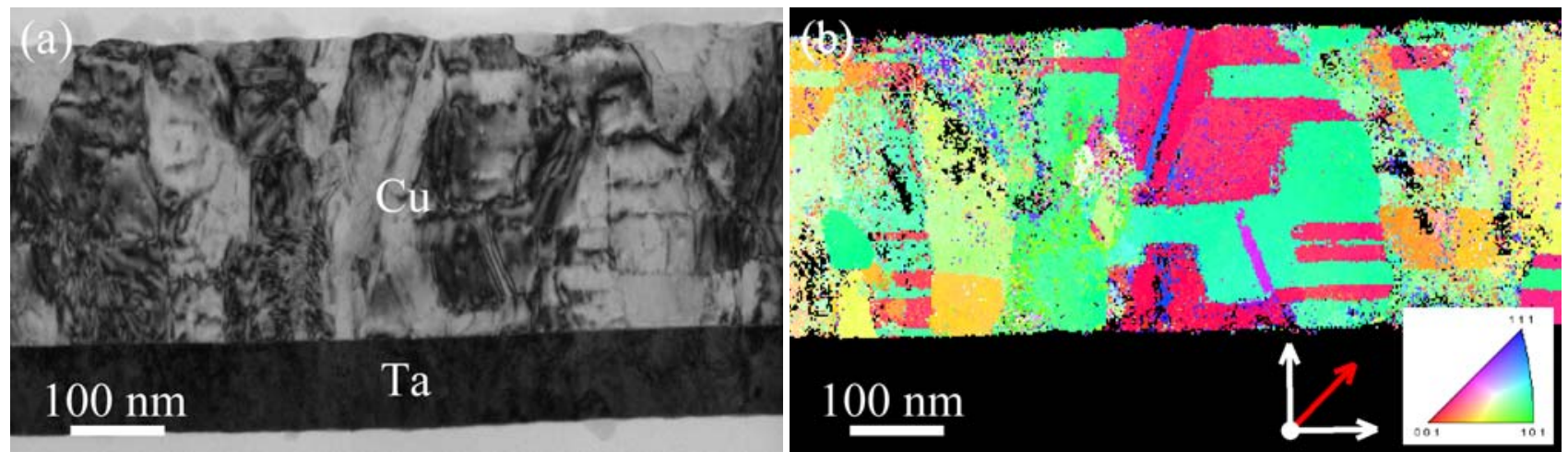

FIG. 1. Cross-sectional TEM BF image of a blanket $\mathrm{Cu}$ film on a Ta diffusion barrier (a) with an IPF maps $45^{\circ}$ inclined to the wafer surface (b) (reprinted with permission from reference 2, copyright [2010], American Institute of Physics).
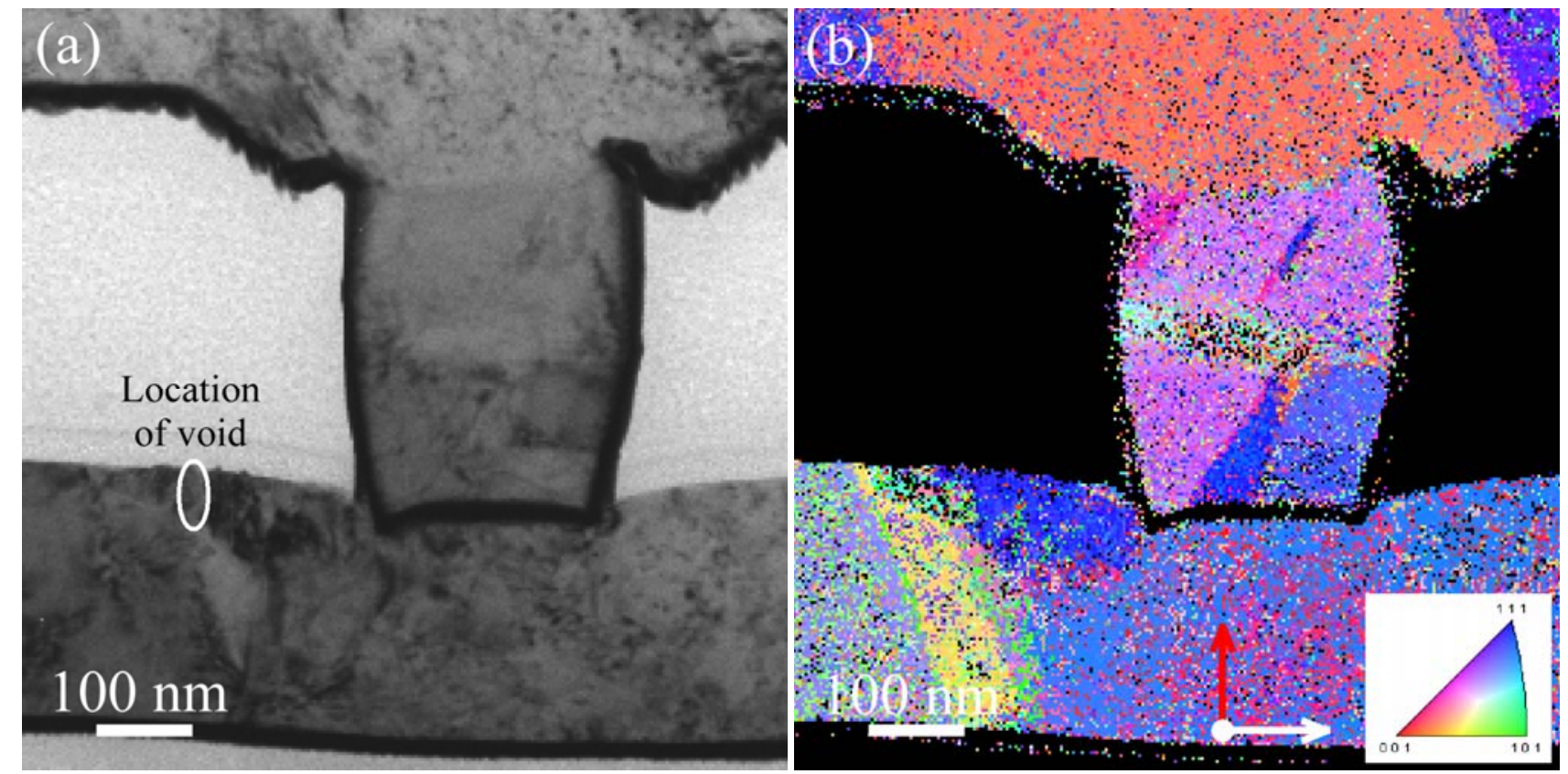

FIG. 2. Cross-sectional TEM BF image of the structure of interest (stress-induced void and surrounding $\mathrm{Cu}$ grains) after reliability test (a) and IPF map perpendicular to the sample surface (b) (reprinted with permission from reference 3, copyright [2009], American Institute of Physics). 